VOL. 50 (1994) [205-218]

\title{
OPTIMALITY CONDITIONS FOR MULTIOBJECTIVE AND NONSMOOTH MINIMISATION IN ABSTRACT SPACES
}

\author{
L. Coladas, Z. Li and S. Wang
}

\begin{abstract}
In this paper we study optimality conditions for an efficient solution in various senses of a general multiobjective optimisation problem in abstract spaces. We utilise properties of the Clarke's generalised differential and properties of a conesubconvexlike function to derive a few necessary and/or sufficient conditions for a feasible solution to be a weak minimum (a minimum, a strong minimum or a proper minimum) of the vector optimisation problem. The results in this paper are extensions and refinements of some known results in vector optimisation.
\end{abstract}

\section{INTRODUCTION}

In the last two decades, much attention has been paid to optimisation problems with multiple objectives because multicriteria decision models can be better applied to real world problems. Several concepts about optimal solutions have been proposed for a multiobjective programming problem since Yu published his well-known paper [15]. See [9] and [12] for a survey of the solution concepts and theory of vector optimisation.

Most of the publications in the field of vector optimisation discuss problems in finite dimensional spaces and assume that the functions involved in the problems are differentiable. But in some situations, we have to solve a nondifferentiable and multiobjective optimisation problem in abstract spaces [6]. Hence, there are requirements of studying nonsmooth and multiobjective optimisation. A few works on optimality conditions for nondifferentiable and multiobjective optimisation in abstract spaces have appeared recently, see [8], [9] and [10]. In this paper, we study a general multiobjective minimisation problem in abstract spaces. Using properties of the Clarke's generalised differential, we give one necessary condition for a feasible solution to be a weak minimum, a minimum, a strong minimum and a proper minimum of the problem respectively. Under a very mild assumption, we prove that the necessary conditions are also sufficient. The

Received 13th October, 1993

Partially supported by NNSFC and MADIS. This paper was prepared during the third author's stay at Departamento de Estadística e Investigación Operativa, Universidad de Santiago de Compostela, Spain. He is very grateful to the financial support for his visit provided by Universidad de Santiago. Names of the authors are given in alphabetical order.

Copyright Clearance Centre, Inc. Serial-fee code: 0004-9729/94 SA2.00+0.00. 
results in this paper are extensions and refinements of some corresponding results in the literature.

We organise this paper as follows. We introduce a few concepts on a solution to the multiobjective optimisation problem, the Clarke's generalised differential and the generalised convexity in the second section. Two lemmas on properties of a pre-invex function and a fundamental optimality condition are proved in Section 2. In Sections 3, 4,5 and 6 , we give a necessary condition and a sufficient condition for a weak minimum, a minimum, a strong minimum and a proper minimum respectively.

\section{Preliminaries}

In this section, we introduce a few notations, definitions and lemmas.

Let $X$ be a real Banach space with norm $\|\cdot\|$ and $Y$ a real locally convex separable topological vector space. We denote the topological dual spaces of $X$ and $Y$ by $X^{*}$ and $Y^{*}$ respectively, which are assumed to be equiped with the weak * topology. Let $\Omega$ be a nonempty subset of $X$ and $K$ a closed convex cone of $Y$. For set $A \subset Y$, its interior and its closure are denoted by int $A$ and $\operatorname{cl} A$ respectively, its dual cone $A^{+}$ and its strict dual cone $A^{s+}$ are respectively defined as

$$
A^{+}=\left\{y^{*} \in Y^{*}:\left\langle y, y^{*}\right\rangle \geqslant 0, \quad \forall y \in A\right\}
$$

and

$$
A^{s+}=\left\{y^{*} \in Y^{*}:\left\langle y, y^{*}\right\rangle>0, \quad \forall y \in A \backslash\{0\}\right\} .
$$

The cone generalised by $A$ and the tangent cone to $A$ at $y \in \operatorname{cl} A$ are defined, respectively as

$$
P(A)=\{\alpha y: \quad y \in A, \alpha \geqslant 0\}
$$

and

$$
T(A, y)=\left\{d \in Y: \exists t_{k}>0 \text { and } y_{k} \in A \text { such that } y_{k} \rightarrow y \text { and } t_{k}\left(y_{k}-y\right) \rightarrow d\right\} .
$$

Let $h$ be a locally Lipschitz function defined on $X$. We denote by $h^{\circ}(x ; v)$ and $\partial^{\circ} h(x)$ the Clarke's generalised directional derivative of $h$ at $x$ in the direction $v$ and the Clarke's generalised subdifferential of $h$ at $x$, respectively.

We quote here some properties of $h^{\circ}(x ; v)$ and $\partial^{\circ} h(x)$. See [3] for a detailed discussion. 
Lemma 2.1. [3, Propositions 2.1.1 and 2.1.2]

(1) $\partial^{\circ} h(x)$ is a nonempty convex weak * compact subset of $X$;

(2) $h^{\circ}(x ; v)=\max \left\{\left\langle v, x^{*}\right\rangle: x^{*} \in \partial^{\circ} h(x)\right\}$;

(3) $h^{\circ}(x ; \cdot)$ is a positively homogeneous, convex and continuous function on $\boldsymbol{X}$

(4) $\partial^{\circ} h(x)=\partial h(x), \forall x \in X$ when $h$ is convex on $X$, where $\partial h(x)$ denotes the subdifferential of $h$ at $x$.

The vector minimisation problem considered in this paper can be formulated as

$$
\text { (VP) } \quad\left\{\begin{array}{l}
\text { V-minimise } f(x) \\
\text { subject to } x \in \Omega
\end{array}\right.
$$

where $f: X \rightarrow Y$ satisfies that $k^{*} f=k^{*} \circ f$ is locally Lipschitz and regular on $\Omega$ for each $k^{*} \in K^{+}, \Omega$ is a nonempty subset of $X$ and $K$ is a closed convex cone in $Y$ with int $K \neq \emptyset$.

The convex cone $K$ defines a partial order on $Y$. We give the concepts of the following five types of solutions to (VP).

DEFINITION 2.1: Let $\bar{x} \in \Omega$.

(1) $\bar{x}$ is called a weak minimum of (VP) if

$$
(\forall x \in \Omega) \quad f(\bar{x})-f(x) \notin \operatorname{int} K ;
$$

(2) $\bar{x}$ is called a minimum of (VP) if

$$
(\forall x \in \Omega) \quad f(\bar{x})-f(x) \notin K \backslash\{0\} ;
$$

(3) $\bar{x}$ is called a strong minimum of (VP) if

$$
(\forall x \in \Omega) \quad f(x)-f(\bar{x}) \in K ;
$$

(4) $\bar{x}$ is called a Borwein's proper minimum of (VP) if

$$
(-K) \cap \operatorname{cl} T(f(\Omega)+K, f(\bar{x}))=\{0\} ;
$$

(5) $\bar{x}$ is called a Benson's proper minimum of (VP) if

$$
(-K) \cap \operatorname{cl} P(f(\Omega)+K-f(\bar{x}))=\{0\} .
$$


A cone $K$ is said to be pointed if $K \cap(-K)=\{0\}$. From the above definition, we can easily prove the following relations.

LEmMa 2.2 .

(1) A Benson's proper minimum $\Longrightarrow$ a Borwein's proper minimum $\Longrightarrow$ a minimum $\Longrightarrow$ a weak minimum;

(2) A strong minimum is a minimum if $K$ is pointed.

Denote $\mathbb{R}_{+}^{p}=\left\{y \in \mathbb{R}^{p}: y_{i} \geqslant 0, i=1,2, \cdots, p\right\}$.

Next we quote the definitions of a cone-pre-invex function, a cone-convexlike function and a cone-subconvexlike function.

Definition 2.2: [14] $f: X \rightarrow Y$ is said to be $K$-pre-invex on $\Omega$ if there exists a function $\eta: \Omega \times \Omega \rightarrow X$ such that for any $x, y \in \Omega$ and $\lambda \in[0,1], y+\lambda \eta(x, y) \in \Omega$ and

$$
\lambda f(x)+(1-\lambda) f(y)-f(y+\lambda \eta(x, y)) \in K .
$$

Definition 2.3. [5] $f: X \rightarrow Y$ is said to be $K$-convexlike on $\Omega$ if for any $x, y \in \Omega$ and $\lambda \in[0,1]$ there exists a $z \in \Omega$ such that

$$
\lambda f(x)+(1-\lambda) f(y)-f(z) \in K .
$$

Definition 2.4: [7] $f: X \rightarrow Y$ is said to be $K$-subconvexlike on $\Omega$ if there exists $\theta \in$ int $K$ such that for any $x, y \in \Omega, \lambda \in[0,1]$ and $\varepsilon>0$ we can find a $z \in \Omega$ satisfying

$$
\varepsilon \theta+\lambda f(x)+(1-\lambda) f(y)-f(z) \in K .
$$

By Definitions $2.2-2.4$, we get immediately,

Lemma 2.3. $K$-convexity $\Longrightarrow K$-pre-invexity $\Longrightarrow K$-convex-likeness $\Longrightarrow$ $K$-subconvexlikeness.

However, it should be mentioned that the converse of the conclusions in the above lemma is not true. Three corresponding counterexamples can be found in [14], [5] and [7].

LEMMA 2.4.

(1) If $f$ is $K$-pre-invex on $\Omega$ and $k^{*} \in K^{+}$, then $k^{*} f$ is $\mathbb{R}_{+}$-pre-invex on $\Omega$, where $\mathbb{R}_{+}=\{\alpha \in \mathbb{R}: \alpha \geqslant 0\}$;

(2) If $f$ is $K$-subconvexlike on $\Omega$, then $f(\Omega)+\operatorname{int} K$ is convex.

Lemma 2.4 (1) follows from Definition 2.2 and the definition of $K^{+}$; see [8] for a proof of Lemma 2.4(2). 
LEMMA 2.5. [8, Theorem 1] If $f$ is $K$-subconvexlike on $\Omega$, then exactly one of the following holds:

$$
\begin{gathered}
(\exists x \in \Omega)-f(x) \in \operatorname{int} K \\
\left(\exists k^{*} \in K^{+} \backslash\{0\}\right) \quad\left(k^{*} f\right)(\Omega) \subset \mathbb{R}_{+} .
\end{gathered}
$$

Let $x \in \Omega$. The cone of all feasible directions to $\Omega$ at $x$ is defined as

$$
F(\Omega, x)=\left\{v \in X:\left(\exists t_{0}>0\right)\left(\forall t \in\left[0, t_{0}\right]\right) x+t v \in \Omega\right\} .
$$

It is obvious that if $\Omega$ is convex, then

$$
F(\Omega, x)=\{t(y-x): y \in \Omega, t \geqslant 0\}=P(\Omega-x)
$$

which is a convex cone.

LemMA 2.6. Suppose that $h: X \rightarrow \mathbb{R}$ is locally Lipschitz and regular on $\Omega$. If $h$ is $\mathbb{R}_{+}$-pre-invex on $\Omega$, then there exists an $\eta: \Omega \times \Omega \rightarrow X$ such that for any $x, y \in \Omega, \eta(y, x) \in F(\Omega, x)$ and

$$
h(y)-h(x) \geqslant\left\langle\eta(y, x), x^{*}\right\rangle, \quad \forall x^{*} \in \partial^{\circ} h(x) .
$$

Proof: Assume that $h$ is $\mathbb{R}_{+}$-pre-invex on $\Omega$. By Definition 2.2, we can find an $\eta: \Omega \times \Omega \rightarrow X$ such that for any $x, y \in \Omega$ and $\lambda \in[0,1], x+\lambda \eta(y, x) \in \Omega$ and

$$
\lambda h(y)+(1-\lambda) h(x)-h(x+\lambda \eta(y, x)) \geqslant 0 .
$$

Hence, $\eta(y, x) \in F(\Omega, x)$ and

$$
\lambda(h(y)-h(x)) \geqslant h(x+\lambda \eta(y, x))-h(x) .
$$

Dividing the above inequality by $\lambda>0$ and then letting $\lambda \downarrow 0$, we get

$$
h(y)-h(x) \geqslant h^{\prime}(x ; \eta(y, x)) .
$$

Because $h$ is regular on $\Omega$,

$$
h^{\prime}(x ; \eta(y, x))=h^{\circ}(x ; \eta(y, x)) \geqslant\left\langle\eta(y, x), x^{*}\right\rangle, \forall x^{*} \in \partial^{\circ} h(x) .
$$

Therefore,

$$
h(y)-h(x) \geqslant\left\langle\eta(y, x), x^{*}\right\rangle, \quad \forall x^{*} \in \partial^{\circ} h(x) .
$$

This completes the proof.

It should be mentioned that (2.12) is a generalised version of the invexity related to a definition

$$
f(y)-f(x) \geqslant f^{0}(x ; \eta(y, x))
$$

given in [4].

Our next lemma generalises one of the main results in [11]. 
Lemma 2.7. Suppose that $h: X \rightarrow \mathbb{R}$ is locally Lipschitz and regular on $\Omega$. Then

(1) $h$ gets a minimum over $\Omega$ at $x$ if $h$ is $\mathbb{R}_{+}-$pre-invex on $\Omega$ and $\partial^{\circ} h(x) \cap$ $F(\Omega, x)^{+} \neq \emptyset$;

(2) $\partial^{\circ} h(x) \cap F(\Omega, x)^{+} \neq \emptyset$ if $\Omega$ is convex and if $h$ gets a minimum over $\Omega$ at $x$.

Proof: First assume that $h$ is $\mathbb{R}_{+}$-pre-invex on $\Omega$ and $\partial^{\circ} h(x) \cap F(\Omega, x)^{+} \neq \emptyset$, but $x$ is not a minimum of $h$ over $\Omega$. Thus there exists a $y \in \Omega$ such that

$$
h(y)-h(x)<0
$$

Let

$$
x^{*} \in \partial^{\circ} h(x) \cap F(\Omega, x)^{+}
$$

Since $h$ is $\mathbb{R}_{+}$-pre-invex on $\Omega$, by Lemma 2.6 , there exists an $\eta: \Omega \times \Omega \rightarrow X$ such that $\eta(y, x) \in F(\Omega, x)$ and

$$
h(y)-h(x) \geqslant\left\langle\eta(y, x), x^{*}\right\rangle \geqslant 0
$$

This contradicts (2.17). Hence, $x$ is a minimum of $h$ over $\Omega$.

Next we prove conclusion (2) in this lemma. Assume that $\Omega$ is convex and $h$ gets a minimum over $\Omega$ at $x$, but $\partial^{\circ} h(x) \cap F(\Omega, x)^{+}=\emptyset$. By Lemma 2.1 and the definition of a dual cone, $\partial^{\circ} h(x)$ is a weak * compact convex set and $F(\Omega, x)^{+}$is a closed convex cone. By the separation theorem of two convex set [1], we can find an $x^{\circ} \in X$ such that

$$
\left\langle x^{\circ}, y\right\rangle \geqslant 0, \forall y \in F(\Omega, x)^{+}
$$

and

$$
\left\langle x^{\circ}, x^{*}\right\rangle<0, \forall x^{*} \in \partial^{\circ} h(x)
$$

By (2.11), $F(\Omega, x)$ is a convex cone since $\Omega$ is convex. So we have $x^{\circ} \in\left((F(\Omega, x))^{+}\right)^{+}=$ $\operatorname{cl} F(\Omega, x)$. Hence, there exist $x^{k} \in F(\Omega, x), k=1,2, \cdots$, such that $x^{k} \rightarrow x^{\circ}$ as $k \rightarrow \infty$. From the definition of $F(\Omega, x)$, for each $k$, we can find a $t_{k}>0$ such that

$$
x+t x^{k} \in \Omega, \quad \forall t \in\left[0, t_{k}\right] .
$$

Noting that $x$ is a minimum of $h$ over $\Omega$, we have

$$
h\left(x+t x^{k}\right)-h(x) \geqslant 0, \quad \forall t \in\left[0, t_{k}\right]
$$


Since $h$ is regular over $\mathrm{X}$,

$$
h^{\circ}\left(x ; x^{k}\right)=h^{\prime}\left(x ; x^{k}\right) \geqslant 0, k=1,2, \cdots
$$

By Lemma $2.1, h^{\circ}(x ; \cdot)$ is continuous. Letting $k \rightarrow \infty$, we get

$$
h^{\circ}\left(x ; x^{\circ}\right) \geqslant 0 \text {. }
$$

However, (2.21) implies

$$
h^{\circ}\left(x ; x^{\circ}\right)=\max \left\{\left\langle x^{\circ}, x^{*}\right\rangle: x^{*} \in \partial^{\circ} h(x)\right\}<0
$$

which contradicts (2.25). Hence, $\partial^{\circ} h(x) \cap F(\Omega, x)^{+} \neq \emptyset$. This completes the proof.

\section{Weak Minima}

For a weak minimum to the vector minimisation problem (VP), we have a sufficient condition and a necessary condition as follows.

THEOREM 3.1. If $f$ is $K$-pre-invex on $\Omega$ and if there exists a $k^{*} \in K^{+} \backslash\{0\}$ such that $\partial^{\circ}\left(k^{*} f\right)(x) \cap F(\Omega, x)^{+} \neq \emptyset$, then $x$ is a weak minimum of $(V P)$.

Proof: Suppose that $f$ is $K$-pre-invex on $\Omega$ and that there exists $k^{*} \in K^{+} \backslash\{0\}$ such that $\partial^{\circ}\left(k^{*} f\right)(x) \cap F(\Omega, x)^{+} \neq \emptyset$. By Lemma 2.4, $k^{*} f$ is $\mathbb{R}_{+}$-pre-invex on $\Omega$. From the condition $\partial^{\circ}\left(k^{*} f\right)(x) \cap F(\Omega, x)^{+} \neq \emptyset$ and Lemma 2.7(1),

$$
\left\langle f(x)-f(y), k^{*}\right\rangle=k^{*} f(x)-k^{*} f(y) \leqslant 0, \quad \forall y \in \Omega .
$$

Hence, there exists no $y \in \Omega$ such that

$$
f(x)-f(y) \in \operatorname{int} K
$$

because $k^{*} \in K^{+} \backslash\{0\}$ implies that $\left\langle k, k^{*}\right\rangle>0, \forall k \in$ int $K$. Therefore, $x$ is a weak minimum to (VP).

THEOREM 3.2. Suppose that $\Omega$ is convex and that $f$ is $K$-subconvexlike on $\Omega$. If $x$ is a weak minimum of (VP), then there exists a $k^{*} \in K^{+} \backslash\{0\}$ such that $\partial^{\circ}\left(k^{*} f\right)(x) \cap F(\Omega, x)^{+} \neq \emptyset$.

Proof: Define $F: X \rightarrow Y$ as

$$
F(y)=f(y)-f(x), \quad y \in X .
$$

Since $f$ is $K$-subconvexlike on $\Omega, F$ is $K$-subconvexlike on $\Omega$. Assume that $x$ is a weak minimum of (VP). By Definition 2.1(1), the system

$$
-F(y) \in \text { int } K, \quad y \in \Omega
$$


has no solution. According to Lemma 2.5, there exists a $k^{*} \in K^{+} \backslash\{0\}$ such that

$$
k^{*} F(y) \geqslant 0, \quad \forall y \in \Omega
$$

that is,

$$
k^{*} f(y) \geqslant k^{*} f(x), \quad \forall y \in \Omega .
$$

Using Lemma 2.7(2), we have

$$
\partial^{\circ}\left(k^{*} f\right)(x) \cap F(\Omega, x)^{+} \neq \emptyset .
$$

The proof is completed.

From Lemma 2.3, Theorems 3.1 and 3.2, we can immediately get the following corollary.

CoRollary 3.1. Suppose that $\Omega$ is convex and that $f$ is $K$-pre-invex on $\Omega$. Then $x$ is a weak minimum of (VP) if and only if there exists a $k^{*} \in K^{+} \backslash\{0\}$ such that $\partial^{\circ}\left(k^{*} f\right)(x) \cap F(\Omega, x)^{+} \neq \emptyset$.

The above corollary generalises $[13$, Theorem 2.1].

\section{Minima}

A sufficient condition and a necessary condition for a minimum of (VP) will be given in this section.

Theorem 4.1. Let $K$ be pointed and $x \in \Omega$. If $f$ is $K$-pre-invex on $\Omega$ and if there exists a $k^{*} \in K^{+} \backslash\{0\}$ such that $\partial^{\circ}\left(k^{*} f\right)(x) \cap F(\Omega, x)^{\jmath+} \neq \emptyset$, then $x$ is a minimum of (VP).

Proof: Suppose that $f$ is $K$-pre-invex on $\Omega$ and that there exists a $k^{*} \in K^{+} \backslash\{0\}$ such that $\partial^{\circ}\left(k^{*} f\right)(x) \cap F(\Omega, x)^{*+} \neq \emptyset$. Assume that $x$ is not a minimum of (VP). Thus there exists a $y \in \Omega$ such that

$$
f(x)-f(y) \in K \backslash\{0\} .
$$

Since $f$ is $K$-pre-invex on $\Omega$, there exists an $\eta: \Omega \times \Omega \rightarrow X$ such that for any $\lambda \in(0,1), x+\lambda \eta(y, x) \in \Omega$ and

$$
\lambda f(y)+(1-\lambda) f(x)-f(x+\lambda \eta(y, x)) \in K .
$$

We can rewrite (4.2) as

$$
\lambda(f(y)-f(x))+f(x)-f(x+\lambda \eta(y, x)) \in K .
$$


Because $K$ is pointed, by (4.1) and (4.3),

$$
\begin{array}{r}
f(x)-f(x+\lambda \eta(y, x))=\lambda(f(x)-f(y))+[\lambda(f(y)-f(x))+f(x)-f(x+\lambda \eta(y, x))] \\
\in K \backslash\{0\}+K \subset K \backslash\{0\} .
\end{array}
$$

Hence, $\eta(y, x) \neq 0$. By the $K$-pre-invexity of $f$ on $\Omega, k^{*} \in K^{+} \backslash\{0\}$ and Lemma 2.4, $k^{*} f$ is $\mathbb{R}_{+}$-pre-invex on $\Omega$. Hence, from Lemma 2.6, $\eta(y, x) \in F(\Omega, x) \backslash\{0\}$ and

$$
k^{*} f(y)-k^{*} f(x) \geqslant\left\langle\eta(y, x), x^{*}\right\rangle, \quad \forall x^{*} \in \partial^{\circ}\left(k^{*} f\right)(x) .
$$

In particular, if we choose $x^{*} \in \partial^{\circ}\left(k^{*} f\right)(x) \cap F(\Omega, x)^{a+}$, then

$$
\left\langle\eta(y, x), x^{*}\right\rangle>0 .
$$

So, from (4.5), we get

$$
k^{*} f(y)-k^{*} f(x)>0
$$

But (4.1) and $k^{*} \in K^{+}$imply that

$$
k^{*} f(x)-k^{*} f(y) \geqslant 0
$$

which contradicts (4.7). Therefore, $x$ is a minimum of (VP).

From the above theorem, we have the following corollary.

CoROLlaRY 4.1. Let $x \in \Omega, Y=\mathbb{R}^{n}, K=\mathbb{R}_{+}^{n}$ and $f=\left(f_{1}, \cdots, f_{n}\right)$. If $f$ is $\mathbb{R}_{+}^{n}$-pre-invex on $\Omega$ and if there exists an $i$ such that $\partial^{\circ} f_{i}(x) \cap F(\Omega, x)^{\Delta+} \neq \emptyset$, then $x$ is a minimum of (VP).

This corollary can be viewed as an extension of [13, Theorem 3.1].

THEOREM 4.2. Suppose that $\Omega$ is convex and that $f$ is $K$-subconvexlike on $\Omega$. If $x$ is a minimum of (VP), then there exists a $k^{*} \in K^{+} \backslash\{0\}$ such that $\partial^{\circ}\left(k^{*} f\right)(x) \cap$ $F(\Omega, x)^{+} \neq 0$.

Proof: This follows directly from Lemma 2.2 and Theorem 3.2.

\section{Strong Minima}

For a strong minimum of (VP), we have the following analogues of Theorems 3.1 and 3.2 and Corollary 3.1 . 
ThEOREM 5.1. Suppose that $f$ is $K$-pre-invex on $\Omega$. If $x \in \Omega$ and $\partial^{\circ}\left(k^{*} f\right)(x) \cap F(\Omega, x)^{+} \neq \emptyset$ for each $k^{*} \in K^{+}$, then $x$ is a strong minimum of (VP).

Proof: Assume that $x \in \Omega$ and $\partial^{\circ}\left(k^{*} f\right)(x) \cap F(\Omega, x)^{+} \neq \emptyset$ for all $k^{*} \in K$. Thus for each $k^{*} \in K^{+}$, there exists

$$
x^{*}=x^{*}\left(k^{*}\right) \in \partial^{\circ}\left(k^{*} f\right)(x) \cap F(\Omega, x)^{+} .
$$

By Lemma 2.4(1) and the $K$-pre-invexity of $f, k^{*} f$ is $K$-pre-invex on $\Omega$. By Lemma 2.6, there exists an $\eta: \Omega \times \Omega \rightarrow X$ such that for any $y \in \Omega, \eta(y, x) \in F(\Omega, x)$ and

$$
k^{*} f(y)-k^{*} f(x) \geqslant\left\langle\eta(y, x), x^{*}\right\rangle \geqslant 0
$$

So,

$$
\left\langle f(y)-f(x), k^{*}\right\rangle=k^{*} f(y)-k^{*} f(x) \geqslant 0, \forall k^{*} \in K^{+}, \forall y \in \Omega .
$$

Hence,

$$
f(y)-f(x) \in\left(K^{+}\right)^{+}=\mathrm{cl} K=K, \quad \forall y \in \Omega .
$$

This implies that $x$ is a strong minimum of (VP).

THEOREM 5.2. Suppose that $\Omega$ is convex. If $x$ is a strong minimum of (VP), then

$$
\partial^{\circ}\left(k^{*} f\right)(x) \cap F(\Omega, x)^{+} \neq \emptyset, \quad \forall k^{*} \in K^{+} .
$$

Proof: Assume that $x$ is a strong minimum of (VP). Thus

$$
f(y)-f(x) \in K, \quad \forall y \in \Omega
$$

For each $k^{*} \in K^{+}$,

$$
k^{*} f(y)-k^{*} f(x)=\left\langle f(y)-f(x), k^{*}\right\rangle \geqslant 0, \quad \forall y \in \Omega .
$$

By Lemma 2.7(2), we have

$$
\partial^{\circ}\left(k^{*} f\right)(x) \cap F(\Omega, x)^{+} \neq \emptyset .
$$

The proof is completed.

From Theorems 5.1 and 5.2, we have the following corollary.

Corollary 5.1. Suppose that $\Omega$ is convex and that $f$ is $K$-pre-invex on $\Omega$. Then $x \in \Omega$ is a strong minimum of (VP) if and only if $\partial^{\circ}\left(k^{*} f\right)(x) \cap F(\Omega, x)^{+} \neq$ $\emptyset, \forall k \in K^{+}$.

This corollary generalises [13, Theorem 4.1]. 


\section{Proper Minima}

A necessary condition and a sufficient condition for a proper minimum are proved in this section.

We first give three propositions.

Proposition 6.1. Let $k^{*} \in K^{\text {st }}$. If $x$ is optimal to

$$
\left(P\left(k^{*}\right)\right) \quad \begin{cases}\text { minimise } & k^{*} f(x) \\ \text { subject to } & x \in \Omega\end{cases}
$$

then $x$ is a Benson's proper minimum of (VP).

Proof: Let $h \in P(f(\Omega+K-f(x)))$. Thus there exist $\alpha \geqslant 0, y \in \Omega$ and $k \in K$ such that

$$
h=\alpha(f(y)+k-f(x)) .
$$

If $x$ is optimal to $\left(\mathrm{P}\left(k^{*}\right)\right)$, because $k^{*} \in K^{*+} \subset K^{+}$,

$$
k^{*} h=\alpha\left(k^{*} f(y)-k^{*} f(x)+k^{*}(k)\right) \geqslant \alpha k^{*}(k) \geqslant 0 .
$$

Hence,

$$
k^{*} h \geqslant 0, \quad \forall h \in P(f(\Omega)+K-f(x)) .
$$

It follows that

$$
k^{*} h \geqslant 0, \quad \forall h \in \operatorname{cl} P(f(\Omega)+K-f(x)) .
$$

Therefore,

$$
(-K) \cap \operatorname{cl} P(f(\Omega+K-f(x)))=\{0\} .
$$

By Definition 2.1(5), $x$ is a Benson's proper minimum of (VP).

Proposition 6.2. Suppose that $f$ is $K$-subconvexlike on $\Omega$. If $x$ is a Borwein's proper minimum of (VP), then there exists a $k^{*} \in K^{\text {s+ }}$ such that $x$ is optimal to $\left(P\left(k^{*}\right)\right)$.

Proof: Assume that $x$ is a Borwein's proper minimum of (VP). Thus,

$$
(-K) \cap \operatorname{cl} T(f(\Omega)+K, f(x))=\{0\} .
$$

Hence,

$$
(-\operatorname{int} K) \cap \operatorname{cl} T(f(\Omega)+K, f(x))=\emptyset
$$


By Lemma 2.4 and the $K$-subconvexlikeness of $f, f(\Omega)+$ int $K$ is convex. It can be shown that in this case,

$$
f(\Omega)+\operatorname{int} K-f(x) \subset \operatorname{cl} T(f(\Omega)+\operatorname{int} K, f(x))
$$

and $\operatorname{cl} T(f(\Omega)+\operatorname{int} K, f(x))$ is a convex cone. By (6.9) and the separation theorem of two convex sets [1, Theorem 1.11], we have a $k^{*} \in K^{s+}$ satisfying

$$
k^{*} \in(\operatorname{cl} T(f(\Omega)+\operatorname{int} K, f(x)))^{+} .
$$

By (6.8) and (6.9),

$$
k^{*}(f(y)+t k-f(x)) \geqslant 0, \quad \forall y \in \Omega, \forall k \in \operatorname{int} K, \forall t>0 .
$$

Letting $k \in$ int $K$ be fixed and letting $t \downarrow 0$, we get

$$
k^{*}(f(y)-f(x)) \geqslant 0, \quad \forall y \in \Omega .
$$

Therefore, $x$ is optimal to $\left(\mathrm{P}\left(k^{*}\right)\right)$. The proof is completed.

Proposition 6.3. Suppose that $f$ is $K$-subconvexlike on $\Omega$. The following statements are equivalent:

(1) $x$ is a Borwein's proper minimum of (VP);

(2) $x$ is a Benson's proper minimum of (VP);

(3) $x$ is optimal to $\left(P\left(k^{*}\right)\right)$ for some $k^{*} \in K^{s+}$.

Proof: It follows direcly from Propositions 6.1 and 6.2 and the observation that a Benson's proper minimum of (VP) is always a Borwein's proper minimum of (VP).

By Proposition 6.3, we know that a Benson's proper minimum is equivalent to a Borwein's proper minimum if $f$ is $K$-subconvexlike on $\Omega$. In this case, we simply call a proper minimum in the sense of Benson or in the sense of Borwein "a proper minimum". Propositions 6.1, 6.2 and 6.3 are generalisations of [2, Theorem 1], [2, Theorem 2] and [12, Theorems 3.1.1 and 3.4.2], respectively.

Now we can give a necessary condition and a sufficient condition for a proper minimum of (VP).

THeOREM 6.1. Suppose that $f$ is $K$-pre-invex on $\Omega, K$ is pointed and $x \in \Omega$. If there exists a $k^{*} \in K^{++}$such that $\partial^{\circ}\left(k^{*} f\right)(x) \cap F(\Omega, x)^{+} \neq \emptyset$, then $x$ is a proper minimum of (VP).

Proof: Assume that there exists a $k^{*} \in K^{\circ+}$ such that $\partial^{\circ}\left(k^{*} f\right)(x) \cap F(\Omega, x)^{+} \neq$ $\emptyset$. By the $K$-pre-invexity of $f$ and Lemma 2.4(1), $k^{*} f$ is $\mathbb{R}_{+}$-pre-invex on $\Omega$. By Lemma 2.7(1),

$$
k^{*} f(x) \leqslant k^{*} f(y), \quad \forall y \in \Omega
$$


Hence, $x$ is an optimal solution to $\left(\mathrm{P}\left(k^{*}\right)\right)$. But since $k^{*} \in K^{\star+}$, by Lemma 2.3 and Proposition $6.3, x$ is a proper minimum of (VP).

Theorem 6.2. Suppose that $\Omega$ is convex and that $f$ is $K$-subconvexlike on $\Omega$. If $x$ is a proper minimum of (VP), then there exists a $k^{*} \in K^{\circ+}$ such that

$$
\partial^{\circ}\left(k^{*} f\right)(x) \cap F(\Omega, x)^{+} \neq \emptyset
$$

Proof: Assume that $x$ is a proper minimum of (VP). By Proposition 6.3, there exists a $k^{*} \in K^{s+}$ such that $x$ is optimal to $\left(\mathrm{P}\left(k^{*}\right)\right)$. Noting Lemma 2.7(2), we have

$$
\partial^{\circ}\left(k^{*} f\right)(x) \cap F(\Omega, x)^{+} \neq 0
$$

This completes the proof.

Because $K$-pre-invexity implies $K$-subconvexlikeness, we get the following corollary immediately.

Corollary 6.1. Suppose that $\Omega$ is convex and that $f$ is $K$-pre-invex on $\Omega$. $x$ is a proper minimum of (VP) if and only if $x \in \Omega$ and there exists a $k^{*} \in K^{s+}$ such that

$$
\partial^{\circ}\left(k^{*} f\right)(x) \cap F(\Omega, x)^{+} \neq \emptyset
$$

\section{REFERENCES}

[1] V. Barbu and Th. Precupanu, Convexity and optimization in Banach spaces (Sijthoff and Noordhoff International Publishers, Holland, 1978).

[2] J.M. Borwein, 'Proper efficient points for maximizations with respect to cones', SIAM J. Control Optim. 15 (1977), 57-63.

[3] F.H. Clarke, Optimization and nonsmooth analysis (Wiley and Sons, New York, 1983).

[4] B.D. Craven, 'Nondifferentiable optimization by smooth approximations', Optimization 17 (1986), 3-17.

[5] T. Jahn, 'Scalarization in multiobjective optimization', in Mathematics of Multiobjective Optimization, (P. Serafini, Editor) (Springer-Verlag, Berlin, Heidelberg, New York, 1985), pp. 45-88.

[6] J. Jahn, Mathematical vector optimization in partially ordered linear apaces (Verlag Peter Lang, Frankfurt am Main, 1986).

[7] V. Jeyakumar, 'A generalization of minimax theorem of Fan via a theorem of the alternative', J. Optim. Theory Appl. 48 (1986), 525-533.

[8] Z.M. Li, 'Generalized Kuhn-Tucker conditions of the vector extremum problem in the linear topological spaces', J. Systems Sci. Math. Sci. 10 (1990), 78-83.

[9] D.T. Luc, Theory of vector optimization (Springer-Verlag, Berlin, Heidelberg, New York, 1989). 
[10] M. Minami, 'Weak Pareto-optimal necessary conditions in a nondifferentiable multiobjective program on a Banach space', J. Optim. Theory Appl. 41 (1983), 451-461.

[11] B. Pshenichnyi, Necessary conditions for an extremum (Marcel Dekker, New York, 1967).

[12] Y. Sawaragi, H. Nakayama and T. Tanino, Theory of multiobjective optimization (Academic Press, New York, 1985).

[13] C. Swartz, 'Pshenichnyi's theorem for vector minimization', J. Optim. Theory Appl. 53 (1987), 309-317.

[14] T. Weir and V. Jeyakumar, 'A class of nonconvex functions and mathematical programming', Bull. Austral. Math. Soc. 38 (1988), 177-189.

[15] P.L. Yu, Cone convexity, cone extreme points, and nondominated solutions in decision problems with multiobjectives J. Optim. Theory Appl. 14 (1974), 319-377.

Departamento de Estadística e Investigación Operativa

Universidad de Santiago de Compostela

15771 Santiago de Compostela

Spain

Institute of Systems Science

Academia Sinica

Beijing 100080

China

\author{
Department of Mathematics \\ University of Inner Mongolia \\ Hohhot 010021 \\ China
}

